\title{
Histopathological Effects of 2,4-Dichlorophenoxyacetic Acid on Sprague-Dawley Rats with Special Reference to its Possible Carcinogenicity
}

\author{
Khaled M A Hassanein \\ Department of Pathology and Clinical Pathology, \\ Faculty of Veterinary medicine, Assiut university, Assiut 71526, Egypt \\ Email: kh-hassanien@hotmail.com \\ Received: 01-08-2011, Accepted: 10-08-2011, Published Online: 17-11-2011 \\ doi: 10.5455 /vetworld.2012.24-30
}

\begin{abstract}
This study investigated the toxic effects of 2,4-dichlorophenoxyacetic acid on male Sprague-Dawley rats. In which, 30 rats were divided into three even groups. First group received orally normal saline ( $3 \mathrm{ml} / \mathrm{kg} \mathrm{b.w.)}$ and kept as normal control in parallel to treated groups (5 rats each), second group received orally 2,4-dichlorophenoxyacetic acid (30 mg/kg b.w.) for 2 months and third group received orally 2,4-dichlorophenoxyacetic acid (30 $\mathrm{mg} / \mathrm{kg}$ b.w.) for 6 months. At the end of each treatment, specimens from the liver, kidneys, lungs, testicles, epididymis and brain were obtained and subjected for histopathological examination. After 2 months of treatment with 2,4-dichlorophenoxyacetic acid, the overall changes were milder and ranged from vascular to necrobiotic findings. On the other hand, changes seen after 6 months were severe particularly in the liver and kidney. In the liver, there were focal areas of necrosis and preneoplastic changes in the form eosinophilic preneoplastic foci, oval cell proliferation and spongiotic pericytoma. Kidneys showed glomerular swelling, thickening of the glomerular basement membrane and proliferation of the renal tubular epithelium. Lesions in the other organs included necrosis and sloughing of bronchiolar epithelial lining and mononuclear cellular infiltration in the lungs, necrosis of seminiferous tubular epithelium in the testicles and neuronal degeneration in the brain. In conclusion, 2,4-dichlorophenoxyacetic acid induced deleterious pathological effects on the vital organs including preneoplastic changes in the liver in Sprague-Dawley rats.
\end{abstract}

Keywords: 2,4-dichlorophenoxyacetic acid, herbicides, histopathology, preneoplasia, Sprague-Dawley rats.

\section{Introduction}

Herbicides commonly known as a weed killers are substances used to kill unwanted plants. Selective herbicides kill specific targets while leaving the desired crop relatively unharmed. Of which, 2,4-dichlorophenoxyacetic acid (2,4-D) is a selective systemic phenoxy herbicide that mimics the plant growth regulator indole-3-acetic acid (also known as auxin) and the first herbicide that was registered in the United States in 1946 (Paulino et al., 1994). Nowadays, 2,4-D is used extensively because of its efficacy and low toxicity. The main agricultural uses of 2,4-D include applications to agricultural crops, forests or recreational areas. Subsequently, it may be transported by wind and water into drainage canals, rivers, lakes and oceans (Fargasova, 1994). Animals or humans intoxications with 2,4-D can occur upon exposure to contaminated soil, air, water or crops or after failure to comply with safety instructions (Morgulis et al., 1998).

The acute toxicity of 2,4-D varies depending on its form and type of exposure. Rats subjected to acute intoxication showed ataxia, central nervous system (CNS) depression, muscular weakness and gasping for breaths (Rosso et al., 2000). CNS effects such as general sedation, tenseness, loss of righting reflex, motor incoordination and coma have been described after exposure to large doses of 2,4-D in humans, monkeys, rats, mice, guinea pigs, dogs, cattle, pigs and rabbits (Hess, 1993). The subchronic toxicity affected the kidney (increased kidney weight, histopathological lesions) and the liver (increased liver weight, increased liver enzymes) 
(Gorzinski et al., 1987 and Timchalk, 2004). Chronic toxicity in rats was manifested by decreased body-weight gain, altered organ weights and hematological parameters and other biochemical changes. Chronic toxicity in dogs based on decreased body-weight gain and glucose level with increased creatinine and alanine aminotransferase (ALT), decreased food consumption and brain weight and histopathological changes in the form of pigmentation in tubular epithelium in kidneys of both sexes and pigmentation in the liver sinusoidal lining cells in females (Charles et al., 1996). Moreover, 2,4-D produced a variety of neurotoxic effects following both acute and repeated-dose exposures. One of the most common neurotoxic symptoms caused by 2,4-D exposure was myotonia (Mattson et al., 1997).

2,4-D is absorbed by plant leaves, stems, and roots and is translocated throughout the plant. It causes little harm to grass crops. Unlike auxins, 2,4-D remains at high levels within plant tissues rather than fluctuating as the naturally-occurring hormone. As a result, the plant's transport systems become blocked and destroyed by abnormally fast growth that leads to plant death (Hallenbeck and Cunninghan-Burns, 1985). In animals, the chlorophenoxy acids suppress the synthesis of ribonuclease, uncouple oxidative phosphorylation, demyelinate peripheral nerves and elevate circulating levels of hepatic peroxisomes such as creatinine phosphokinase (CPK), lactate dehydrogenase (LDH) and aspartate aminotransferase (ALT). Skeletal damage as a result of 2,4-D was manifested by increasing the levels of myoglobin and creatin in the urine (Gorzinski et al., 1987). 2,4-D toxicity could be also favor the attack by the reactive oxygen compounds triggering a metabolic stress response reaction resulting in permanent damage (Chinalia et al., 2007). The International Agency for Research on Cancer (IARC) has classified 2,4-D among the phenoxy acid herbicides 4-chloro-2-methylphenoxyacetic acid (MCPA) and 2,4,5-T as class 2B carcinogenpossibly carcinogenic to humans, inadequate evidence for carcinogenicity to animals (IARC, 1987).
This study was built up to investigate the toxic effects of 2,4-D on male Sprague-Dawley rats with special reference to its carcinogenesis.

\section{Materials and Methods}

All experimental procedures in this study were done in accordance with the guidelines of the European Union Council (86/609/EU) for the use of laboratory animals. In which, Thirty Sprague-Dawley (SD) rats aged 2 - 3 months and weighing 150-170 $\mathrm{g}$ were obtained from Helwan Farm for Laboratory Animal Breading, Cairo, Egypt. 2,4-D was obtained from Sigma-Aldrich (USA).

Treatment of rats with 2,4-D: Animals were housed 4 per cage with wood shavings and were maintained under a controlled environment with temperature at $23 \pm 2^{\circ} \mathrm{C}$, relative humidity at $55 \pm$ $5 \%$ and a $12 \mathrm{hr}$ light/dark cycle throughout the experiment. Thirty rats were divided into three even groups. First group received orally normal saline by stomach tube ( $3 \mathrm{ml} / \mathrm{kg} \mathrm{b.w.)} \mathrm{and} \mathrm{kept} \mathrm{as}$ normal control in parallel to treated groups ( 5 rats each), second group received orally 2,4-D 1/10 of $\mathrm{LD}_{50}(30 \mathrm{mg} / \mathrm{kg}$ b.w.) for 2 months and third group received orally 2,4-D (30 mg/kg b.w.) for 6 months.

Histopathology: At the end of each treatment, tissue specimens were obtained from the liver, kidneys, lungs, testicles, epididymis and brain. Specimens were fixed in $10 \%$ neutral buffered formalin, dehydrated in a graded alcohol series, cleared with methyl benzoate and embedded in paraffin wax. Sections of $5 \mu \mathrm{m}$ were cut and stained with haematoxylin and eosin (HE) and Periodic acid-Schiff (PAS) for light microscopic examination (Bancroft et al., 1996). Stained sections were examined under light microscope (Olympus CX31, Japan) and photographed using digital camera (Olympus, Camedia C-5060, Japan).

\section{Results}

Clinical signs and gross Pathology: Along the course of experiment, rats only exhibited slight loss of weight with some degree of disappetite. Grossly, there were congestion in the liver and 


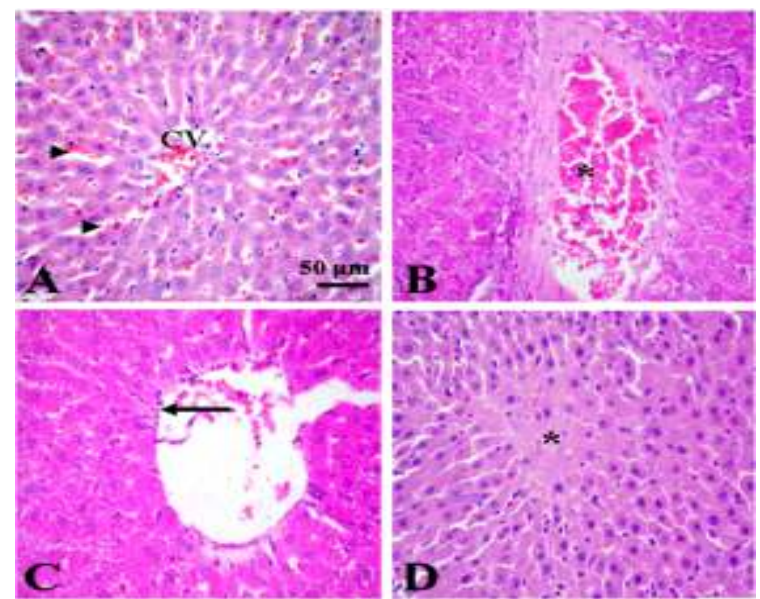

Fig.1 (Above).Representative micrographs for histopathological findings in the liver after 2 month of treatment with 2,4-D. A) Congestion of central vein (CV) and dilatation of the blood sinusoids with blood (arrow heads). B) Congestion of portal vein (asterisk). C) Dilatation of central vein with desquamation of it hepatocytes (asterisk).HE. Fig.2 (Right Side) Representative photomicrographs of histopathologic findings in the liver after 6 month of treatment with 2,4-D. A) Focal Kupffer cell hyperplasia (arrow). B) Oval cell proliferation in the portal area (arrows). C) Oval cell proliferation with development of lumen (bile duct like structure) (arrows). D) Megalocytosis and nuclear atypism (arrow). E) Eosinophilic preneoplastic foci. Note the ballooned cytoplasm and hyperchromasia of the nuclei. F) Spongiotic pericytoma/spongiosis hepatis. HE.

lungs and kidneys appeared pale and swollen. Neither clinical signs nor gross changes were seen in control animals.

\section{Histopathology}

Liver : Histopathological examination of HEstained sections revealed that treatment of rats with 2,4-D for 2 months produced mild vascular and hepatocellular lesions in the liver. Vascular changes were congestion of central and portal veins, dilatation of blood sinusoids with blood and desquamation of the endothelial lining of some blood vessels (Figs.1A-C). Hepatocellular changes were in the form of necrobiotic changes and focal areas of necrosis (Fig. 1D). On the other hand, hepatic lesions were severe after treatment of rats with 2,4-D for 6 months. They consisted of multiple focal areas of Kupffer cell hyperplasia (Fig. 2A). The distinct histopathological findings following treatment of rats with 2,4-D for 6 months was the preneoplastic changes of hepatocytes. Preneoplastic changes was characterized by presence of many oval cells in the portal areas which characterized by small, oval and lightly-stained nuclei surrounded by indistinct cytoplasm. In most cases, the
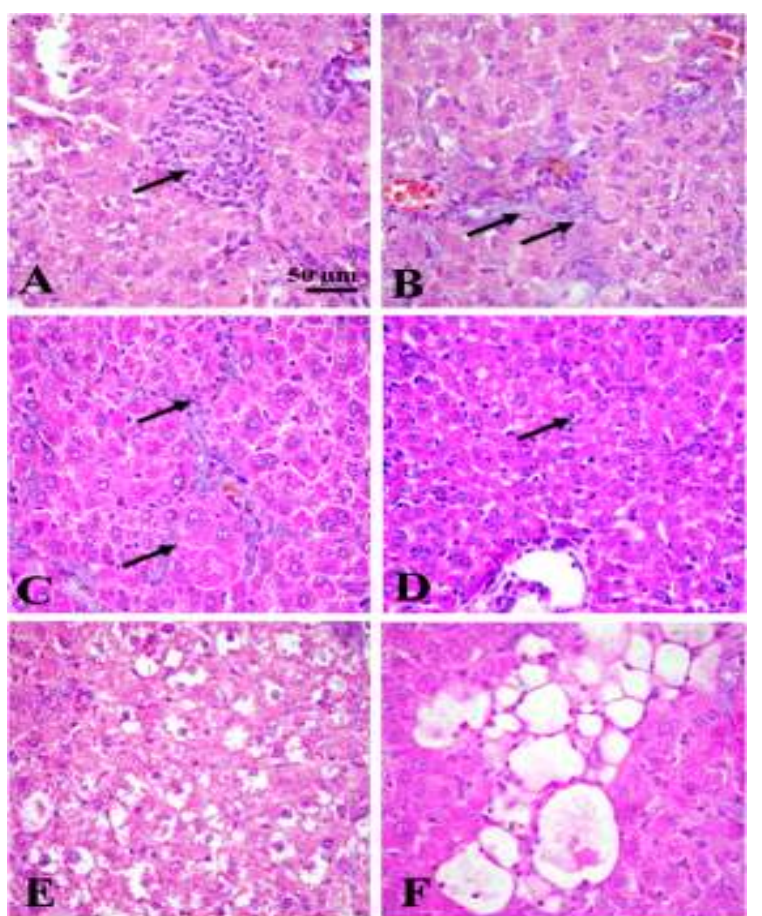

proliferated oval cells were seen in-between the hepatocytes forming bile duct like structures (Figs. 2B,C). Megalocytosis of some hepatocytes and large dysplastic nuclei with a coarse chromatin pattern were observed (Fig. 2D). There were also eosinophilic preneoplastic foci appeared in nine cases out of ten cases. In which, hepatocytes showed clear and ballooned cytoplasm with hyperchromatic nuclei and prominent nucleoli (Fig. 2E). Moreover, there was spongiotic pericytoma/spongiosis hepatis in six treated rats. It is characterized by cyst like multilocular formation with thin wall and elongated nuclei (Fig. 2F).

Kidneys: Treatment of rats with 2,4-D for 2 months resulted in congestion of renal blood vessels, interstitial edema and mononuclear cellular infiltration, glomerular swelling and hyaline droplet degeneration in the renal tubular epithelium (Figs. 3A, B). Rats treated for 6 months exhibited marked glomerular and tubular lesions. Glomeruli appeared swollen as the result of mesangial proliferation and showed thickening of the glomerular basement membrane and Bowman's capsule (Figs. 3C-E). Renal tubular 


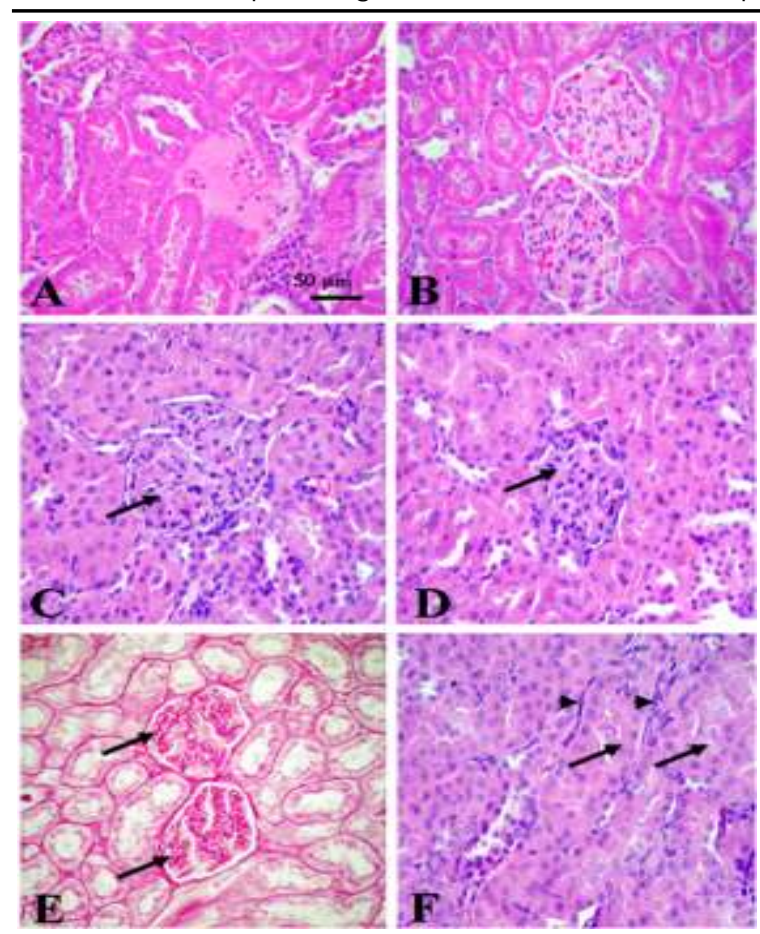

Fig. 3. Representative photomicrographs of histopathologic findings in the kidney after 2 and 6 months of treatment with 2,4-D. A) Kidney showing congestion, interstitial edema and mononuclear cellula infiltration. B) Swollen glomerulus as a result of congestion. C,D Glomerular swelling as a result of proliferation of mesangial cells (arrows). E) Thickening of the glomerular basement membrane and Bowman's capsule (arrows). F) Proliferation of the renal tubular epithelium (arrows) and proliferation of fibroblast in the interstitium (arrow heads) . A, B, C, D, F stained with $\mathrm{HE}$ and $\mathrm{E}$ stained with PAS.

epithelial cells appeared proliferated and some of them had double nuclei and prominent nucleoli. There was also fibroblastic proliferation in the interstitum (Fig. 3F). The incidence of the most common lesions in liver and kidney of rats treated with 2,4-D after 2 and 6 months of treatment was recorded (Table 1).

Other organs: Histopathological examination of other organs including lungs, testicles, epididymis and brain revealed congestion of blood vessels after treatment with 2,4-D for 2 months. After 6 months of treatment, histopatho-logical findings were necrosis and sloughing of the bronchiolar epithelium and peribronchiolar lymphocytic infiltration (Figs. 4A, B). Testicles showed necrosis and sloughing of seminiferous tubular epithelium (Fig. 4C) and epididymis revealed necrobiotic changes of the epithelial lining and mononuclear cellular infiltration in the interstitium (Fig. 4D).

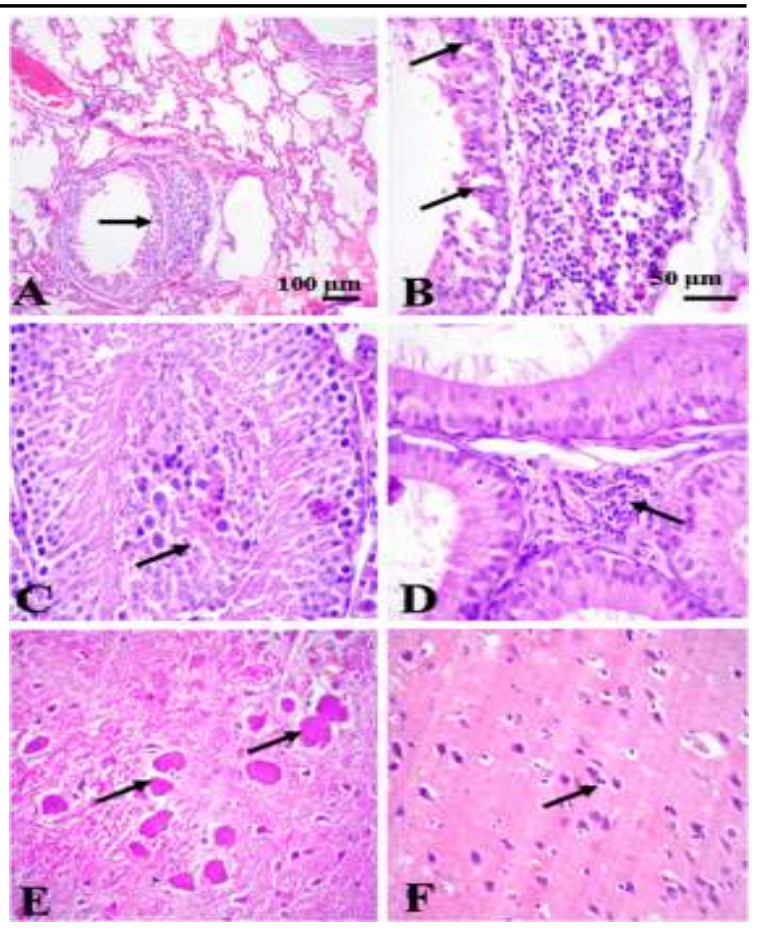

Fig. 4. Representative micrographs for some histopathological changes in lungs, testicles, epididymis and brain of 2,4-D treated animals. A,B) Lung showing necrosis and sloughing of bronchiolar epithelium (arrows) and heavy infiltration of lymphocytes. C) Testicle showing necrosis and sloughing of necrosed cells in the lumen of seminiferous tubule (arrow). D) Mononuclear cell infiltration in the interstitium of epididymis (arrow) E) Ischemic neuronal degeneration and peri-neuronal edema (arrows). F) Microglial reaction (arrow). HE.

Brain showed neuronal degeneration characterized by presence of ghost nuclei. Also, there were perineuronal edema and mild microglial reaction (Fig. 4F).

\section{Discussion}

The herbicide 2,4-D has been used extensively in the modern agriculture and despite its short half-life in soil and aquatic environment, toxicological studies indicated that it has a great potential for undesirable effects. These toxic effects include embryotoxicity, teratogenicity, neurotoxicity, immunotoxicity and hepatoto-xicity (Tuschl and Schwab, 2003 and Chinalia et al., 2007).

Liver is the first target organ for toxicological prospects because of its role in detoxification, biotransformation and excretion of xenobiotics. After enteric uptake of injurious materials, it is the first organ to be exposed to such hazards via the portal circulation (Katzung, 1990). Results 
Table-1. The incidence of the most common lesions in liver and kidney of rat treated with 2,4-D after 2 and 6 months of treatment.

\begin{tabular}{lcc}
\hline Lesions & No. of rats at $\mathbf{2}$ months & No. of rats at6months \\
\hline Liver Lesions & 10 & 2 \\
- Congestion & 8 & 1 \\
- Desquamation of the endothelial lining & 9 & 0 \\
- Focal liver necrosis & 0 & 9 \\
- Focal Kupffer cell hyperplasia & 0 & 9 \\
- Preneoplastic foci & 0 & 9 \\
- Oval cell proliferation & 0 & 9 \\
- Megalocytosis & 0 & 6 \\
- Spongiotic pericytoma & & 0 \\
Kidney lesions: & 10 & 0 \\
- Congestion & 10 & 5 \\
- Edema & 10 & 8 \\
- Glomerular swelling & 0 & 10 \\
- Thickening of glomerular basement membrane & 0 & 10 \\
- Proliferation of the renal epithelium & 0 & \\
- Fibroblast proliferation & 0 & \\
\hline
\end{tabular}

obtained in the present study showed that 2,4-D induced many histopathological changes in the liver of rats included necrobiotic changes, focal areas of necrosis and preneoplastic changes. Gorzinski et al. (1987) reported necrobiotic changes in the form of hepatocellular cytoplasmic swelling and homogeneity in rats received $15 \mathrm{mg} / \mathrm{kg}$ b.w. of 2,4-D per day. Hallenbeck and CunninghanBurns (1985) observed focal necrosis of the hepatocytes in rats following treatment with 2,4-D.

Many herbicides are reported to be carcinogenic in rats. For example, acetochlor and alachlor induced tumors in the nasal turbinates, butachlor induced stomach tumors, and metolachlor causes liver tumors (Coleman et al., 2000). However some studies reported that 2,4-D produced brain tumor in rats and lymphoma in dogs and rats, the carcinogenic potential of 2,4-D remains controversial (Page, 1996). In the present study, treatment of rats with 2,4-D for 6 months produced preneoplastic changes in the form of eosinophilic preneoplastic foci, oval cell proliferation and spongiotic pericytoma. Preneoplastic foci of altered hepatocytes have been known as the earliest emerging distinct phenotypic parenchymal changes indicating carcinogenic response in chemical hepatocarcinogenesis in rats and mice for almost 4 decades and have been discussed as potential end-points in carcinogenicity testing since in the early 1980 (Bortolozzi et al., 2001).

Oval cell proliferation can give rise to preneoplastic foci and nodules that can progress to cancer (Sell and Dunsford, 1989). He et al.
(1994) observed oval cell proliferation following exposure of mice to a single dose of N-nitrosodiethylamine. Moreover, de lima et al. (2008) found that exposure of rats to N-nitrosodiethylamine in dinking water $(135 \mathrm{mg} / \mathrm{l})$ for 16 weeks showed oval cell proliferation which appeared dispersed among mature large hepatocytes or forming small cords of clear cells. The preneoplastic or neoplastic nature of spongiosis hepatis has been considered based on its first description in 1997 in both rats and fish (Couch and Courtney, 1987; Couch, 1991 and Bannasch and Zerban, 1997). Furthermore, Newton et al. (2001) and Spencer et al. (2002) reported that spongiosis hepatis is an indicator of hepatocarcinogenicity after treatment of rats and mice with methylkethylketoxime or propylene glycol monomethyl ether.

Kidney is a potential target for chronic occupational exposure to phenoxy acids as they are inhibitors of chloride channels in renal tubular cells which leads to alterations in the excretion of urinary electrolytes (Boers et al., 2010). Histopathological examination of renal tissues revealed vascular changes such as interstitial edema and congestion of glomerular capillaries after treatment with 2,4-D for 2 months. Treatment of rats for 6 months resulted in mesangial cell proliferation, thickening of the glomerular basement membrane and proliferation of the tubular epithelium. Similarly, it was reported that treatment of rats with 2,4-D produced loss of brush border and hypertrophy 
and karyomegaly of the renal tubular epithelial cells (Paulino et al., 1994 and Timchalk, 2004). Moreover, it was found that 2,4-D produced proliferation of the renal tubular epithelium in rats after 6 months of treatment. Hard et al. (1999) reported that hyperplasia of renal tubular epithelium implies an increase in the number of lining cells inside the tubules i.e. without proliferation beyond the basement membrane.

Pulmonary lesions as the result of treatment with 2,4-D were necrosis of the bronchiolar epithelium and heavy peribronchiolar infiltration of lymphocytes. Similarly, Charles et al. (1996) found that 2,4-D induced subacute to chronic inflammation of the rat lung. Examination of brain tissue revealed that 2,4-D after 6 months induced neuronal degeneration and microglial proliferation. In parallel, Bortolozzi et al. (2001) found that 2,4-D affected monoaminergic terminals, axonal fibers and cell bodies. Moreover, Konjuh et al. (2008) reported that 2,4$\mathrm{D}$ induced a high vulnerability to the herbicide effects on myelinogenesis in pups of Wistar rats exposed through the mother's milk. 2,4-D was also found to affect genital organs as it produced necrosis in the seminiferous tubular epithelium and necrobiotic changes and mononuclear cellular infiltration in the epididymis. In this context, Server et al. (1997) found that 2,4-D reduced sperm counts and increased abnormalities in sperm humans.

In conclusions, 2,4-D adversely affected different organs in rats, most notably the liver and kidney. The prominent lesions in the liver were the preneoplastic changes in the form of preneoplastic foci, oval cell proliferation and spongiotic pericytoma. The characteristic lesions in the kidney were thickening of the glomerular basement membrane and proliferation of the renal tubular cells. Taken together, 2,4-D appeared to be potentially carcinogenic to rat liver.

\section{Acknowledgements}

Authors would like to thank all staff members, technicians and administrative staffs in the Department of Pathology and Clinical Pathology, Faculty of Veterinary Medicine, Assiut University for helping and providing facilities.

\section{Conflict of interest}

Authors declare that they have no conflict of interest.

\section{References}

1. Bancroft T.D., Stevens A. and Turner D.R. (1996). Theory and practice of histological technique. 4th ed. Churchill, Livingston, New York, London, San Francisco, Tokyo.

2. Bannasch P. and Zerban H. (1997). Experimental chemical hepatocarcinogenesis. In: Okuda K. and Tabor E. (eds). Liver Cancer. pp. 213-253, Churchill Livingstone, New York.

3. Boers D., Portengen L., Bas H. Heederik D. and Vermeulen R. (2010). Cause-specific mortality of Dutch chlorophenoxy herbicide manufacturing workers Occup. Environ. Med., 67: 24-31.

4. Bortolozzi A., de Duffard A.M.E., Dajas F., Duffard R. and Silveira R. (2001). Intracerebral Administration of 2,4-Diclorophenoxyacetic Acid Induces Behavioral and Neurochemical Alterations in the Rat Brain. Neurotoxicology, 22: 221-232.

5. Charles J.M., Bond D.M., Jeffries T.K., Y a n o B.L., Stott W.T., Johnson K.A., Cunny H . C ., Wilson R.D. and Bus J.S. (1996). Chronic dietary toxicity/oncogenicity studies on 2,4dihydrophenoxyacetic acid in rodents. Fundam. Appli. Toxicol., 33:166-172.

6. Chinalia F.A., Seleghin M.H. and Correa E.M. (2007). 2,4-D causes, effect and control. Terrestrial and Aquatic Environmental Toxicology, 1 (2): 24-33.

7. Coleman S., Linderman R., Hodgson E. and Rose R.L. (2000). Comparative metabolism of chloroacetamide herbicides and selected metabolites in human and rat liver microsomes. Environ. Health Perspect., 108(12): 1151-1157.

8. Couch J.A. (1991). Spongiosis hepatis: Chemical induction pathogenesis and possible neoplastic fate in a Teleost fish model. Toxicol. Pathol., 19: 237-250.

9. Couch J.A. and Courtney L.A. (1987). Nitrosodiethylamine-induced hepatocarcinogenesis in estuarine sheephead minnow (Cyprinodon variegatus): Neoplasms and related lesions compared with mammalian lesions. J. Natl. Cancer Inst., 79: 297-321.

10. De Lima V.M.R., Oliveira C.P.M.S., Alves V.A.F., Chammas M.C., Oliveira E.P., Stefano J.T., de Mello E.S., Giovanni G.G., Carrilho F.J. 
and Caldwell S.H. (2008). A rodent model of NASH with cirrhosis oval cell proliferation and hepatocellular carcinoma. Journal of Hepatology, 49: 1055-1061.

11. Fargasova A. (1994). Comparative study of plant growth hormones (herbicides) toxicity in various biological subjects. Ectoxicology and Environmental Safety, 29: 359-364.

12. Gorzinski S.J., Kociba R.J., Campbell R.A., Smith F.A., Nolan R.J. and Eisenbrandt DL. (1987). Acute pharmacokinetic and subchronic toxicological studies of 2,4-dichlorophenoxyacetic acid. Fund. Appl. Toxicol., 9: 423-35.

13. Hard G.C., Alden C.L., Bruner R.H., Frith C.H., Lewis R.M., Owen R.A., Krieg K. and Durchfeld-Mayer B. (1999). Proliferative lesions of the kidney and lower urinary tract in rats. In: Guides for Toxicologic Pathology, vol URG-1, Society of Toxicologic Pathologists, Washington DC.

14. Hallenbeck W.P. and Cunninghan-Burns K.M. (1985). Pesticide and Human Health. SpringerVerlag, New York, NY.

15. He X.Y., Smith G.J., Enno A. and Nicholson R.C. (1994). Short-term diethylnitrosamineinduced oval cell responses in three strains of mice. Pathology, 26 (2): 154-160.

16. Hess F.D. (1993). Herbicide effects on plant structure, physiology and biochemistry. In: Pesticide interactions in crop production: Beneficial and deleterious effects. Altman J. ed. CRC Press, Boca Raton, FL.

17. IARC. (1987). Overall evaluations of carcinogenicity: an updating of IARC. Monographs on the Evaluation of Carcinogenic Risks to Humans International Agency for Research on Cancer. Volumes 1-42, pp. 156-160.

18. Katzung B.G. (1990). Basic and clinical pharmacology, 3rd ed, Appleton and lang, connreticut, USA.

19. Konjuh C., Garci`a G., Lo’pez, L., Mari`a A., de Duffard E., Brusco A., Duffard R. (2008). Neonatal Hypomyelination by the Herbicide 2,4-DichlorophenoxyaceticAcid. Chemical and Ultrastructural Studies in Rats. Toxicol. Sci., 104 (2): 332-340.

20. Mattsson J.L, Charles J.M., Yano B.L., Cunny H.C., Wilson R.D., Bus J.S. (1997). Single-dose and chronic dietary neurotoxicity screening studies on 2,4-dichlorophenoxyacetic acid in rats. Fund. Appl. Toxicol., 40: 111-19.

21. Morgulis M.S., Oliveira G.H., Dagli M.L.Z. and Palermo-Neto J. (1998). Acute 2,4 Dichloro-phenoxyacetic Acid Intoxication in Broiler Chicks. Poultry Science, 77: 509-515.

22. Newton P.E., Wooding W.L., Bolte H.F., Derelanko M.J., Hardisty J.F. and Rinehart W.E. (2001). A chronic inhalation toxicity/carcinogenicity study of methylkethylketoxime in rats and mice. Inhal. Toxicol., 13: 1093-1116.

23. Page D.L. (1996). 2,4-D passae EPARegistration muster. Wheat life, 29:9.

24. Paulino C.A., Oliveira G. and Palermo-Neto J. (1994). Acute 2,4-dichlorophenoxyacetic acid intoxication in cattle. Vet. Human Toxicol., 36: 433-436.

25. Paulino C.A., Guerra J.L., Oliveira G. and Palermo-Neto J. (1996). Acute, subchronic and chronic 2,4-dichlorophenoxyacetic acid intoxication in rats. Vet. Human Toxicol., 38: 348-352.

26. Rosso S.B., Ca'ceres O., Evangelista de Duffard A.M., Duffard R. and Quiroga S. (2000). 2,4Dichlorophenoxyacetic acid disrupts the cytoskeleton and disorganizes the golgi apparatus of cultured neurons. Toxicol. Sci., 56: 133-140.

27. Sell S. and Dunsford H.A. (1989). Evidence for the stem origin of hepatocellular carcinoma and cholangiocarcinoma. Am. J. Path., 134: 1347- 1363.

28. Sever L.E., Arbuckle T.E. and Sweeney A. (1997). Reproductive and developmental effects of occupational pesticide exposure: the epidemiological evidence. Ocсup. Med., 12 (2): 305-25.

29. Spencer P.J., Crissman J.W., Stott W.T., Corley R.A., Cieszlak F.S., Schumann A.M. and Hardisty J.F. (2002). Propylene glycol monomethyl ether (PGME). Inhalation toxicity and carcinogenicity in Fischer 344 rats and B6C3F1 mice. Toxicol. Pathol., 30: 570-579.

30. Timchalk C. (2004). Comparative inter-species pharmacokinetics of phenoxyacetic acid herbicides and related organic acids. Evidence that the dog is not a relevant species for evaluation of human health risk. Toxicology, 200: 1-19.

31. Tuschl T. and Schwab C. (2003). Cytotoxic effect of herbicides 2,4- dichlorophenoxyacetic acid HepG2H cells. Food and Chemical Toxicology, 41: 385-393. 